Article

\title{
Modified Roller Coaster Surface in Space
}

\author{
Selçuk BAŞ * (i) and Talat KÖRPINAR * (D) \\ Department of Mathematics, Muş Alparslan University, 49250 Muş, Turkey \\ * Correspondence: slckbs@hotmail.com (S.B.); talatkorpinar@gmail.com (T.K.)
}

Received: 31 December 2018; Accepted: 13 February 2019; Published: 19 February 2019

\begin{abstract}
In this paper, a new modified roller coaster surface according to a modified orthogonal frame is investigated in Euclidean 3-space. In this method, a new modified roller coaster surface is modeled. Both the Gaussian curvature and mean curvature of roller coaster surfaces are investigated. Subsequently, we obtain several characterizations in Euclidean 3-space.
\end{abstract}

Keywords: modified orthogonal frame; euclidean space; roller coaster surfaces

MSC: 53A04; 53A05

\section{Introduction}

In differential geometry, the search for certain classes of surfaces with particular features in three-dimensional Euclidean space, such as ruled and developable surfaces which are 1-parameter smooth families of straight lines, has a significant role in the field of civil engineering and architecture (CAD) [1-6]. Moreover, the work path and workspace of robotic and/or mechanic operations are formed by these spatial curves and surfaces during the generation process [7-9]. One aim of classical differential geometry is the investigation of several classes of surfaces with specific properties, for example, ruled surfaces and revolution surfaces. Thus, it is reasonable to make use of differential geometry to understand these surfaces and spatial curves.

Circular surfaces are also regarded as anessential topic in different areas, such as ruled surfaces. There are two kinds of circular surfaces in investigations: canonical circular surfaces and non-canonical circular surfaces. A canonical circular surface is characterised by a circle crosscut and in which the normal of the circle plane is usually parallel to that of the crosscut. There are several names for the canonical circular surface, including tube surface [10-12], pipe surface [13], and canal surface [14-17]. Moreover, Stasiak et al. [18] designed DNA molecules. A noncanonical circular surface is defined by a non-circular crosscut and the normal of the circle plane is usually not parallel to that of the crosscut. An arbitrary end-effector point $P$ produces a $R R$ circular surface, where $R$ indicates the revolute joint. Hunt [19] studied the algebric equation of a generally $R R$ circular surface and as specified that the diametrical parts along the generally $R R$ circular surface could take the shape of a banana, boomerang, or circle, depending on the values of construction parameters of the $R R$ mechanism. For a general $R R$ circular surface, the spine curve is a circle; however, the normal of a circle plane is generally not parallel with the normal of a crosscut. Cui et al. [20] studied noncanonical circular surfaces.

Roller coasters may appear to be comparatively simple mechanics structures when compared with modern railroad or cars, butthey are excellent illustrations for the use of spacial curve geometries. The geometric definition of the curve of a roller coaster path, beginning with the description of a moving referential, is the path used in this implementation and is described by the sweep of the normal and binormal vectors [21].

A circular surface is a map

$$
M: I \times R / 2 \pi Z \rightarrow \mathrm{E}^{3}
$$


defined by

$$
L(t, \theta)=\beta(t)+r(t)\left(c_{1}(t) \cos \theta+c_{2}(t) \sin \theta\right),
$$

where $\beta, c_{1}, c_{2}: I \rightarrow E^{3}$ and $r: I \rightarrow \mathrm{E}_{>0}$ [22]. The curve $\beta$ a base curve and a double of curves $c_{1}, c_{2}$ a director frame. The standard circles

$$
\theta \mapsto \beta(t)+r\left(c_{1}(t) \cos \theta+c_{2}(t) \sin \theta\right)
$$

are called generating circles. A roller coaster surface is defined as

$$
M(t, \theta)=\beta(t)+r(\cos \theta \mathbf{T}(t)+\sin \theta(\cos \varphi(t) \mathbf{N}(t)+\sin \varphi(t) \mathbf{B}(t))),
$$

where $-\varphi(t)$ is the primitive function of the torsion $\tau(t)$.

Although channel surfaces are well known, roller coaster surfaces have not been studied extensively. Recently, Tuncer et al. $[23,24]$ studied circular surfaces and roller coaster surfaces through theuse of unit quaternions. Abdel-Baky et al. [25] researched local singularities of a space-like circular surface.

In this paper, a modified roller coaster surface according to modified orthogonal frame isinvestigated in Euclidean 3-space. According tothis method, a novel, modified roller coaster is modeled. Both the Gaussian curvature and mean curvature of roller coaster surfaces are investigated. As a result, we obtain severalcharacterizations in the Euclidean 3-space.

\section{Preliminaries}

Let $\beta=\beta(s): I \subset \mathrm{R} \rightarrow \mathrm{E}^{3}$ be an arbitrary curve of arc-length parameter $s$. Let $\{\mathbf{t}(s), \mathbf{n}(s), \mathbf{b}(s)\}$ be the moving Frenet frame throughout $\beta$. As such, the Frenet derivative formulas are given by

$$
\begin{aligned}
& \mathbf{t}^{\prime}(s)=\kappa \mathbf{n}, \\
& \mathbf{n}^{\prime}(s)=-\kappa \mathbf{t}+\tau \mathbf{b}, \\
& \mathbf{b}^{\prime}(s)=-\tau \mathbf{n},
\end{aligned}
$$

where the functions $\mathcal{\kappa}$ and $\tau$ are thecurvature and torsion of $\beta$, respectively,

$$
\begin{aligned}
& g(\mathbf{t}, \mathbf{t})=1, g(\mathbf{n}, \mathbf{n})=1, g(\mathbf{b}, \mathbf{b})=1, \\
& g(\mathbf{t}, \mathbf{n})=g(\mathbf{t}, \mathbf{b})=g(\mathbf{n}, \mathbf{b})=0 .
\end{aligned}
$$

Let $\beta_{i}(i=1,2,3)$ be coordinates in $\mathrm{E}^{3}$. Let $\beta(s)$ be an analytic curve, where s runs through some interval and $\beta(s)$ be an analytic in $s$. We assume that $\beta$ is non-singular, i.e.,

$$
\sum_{i=1}^{3}\left(\frac{d \beta_{i}}{d s}\right)^{2}
$$

is notzero. Therefore, we can parametrize $\beta$ by its arc length $s$. Accordingly, we only consider $\beta$ in the following form:

$$
\beta=\beta(s)=\left(\beta_{1}, \beta_{2}, \beta_{3}\right), s \in I,
$$

where $\beta(s)$ is analytic in $s$. We assume that the curvature function has discrete zero points or $\mathcal{K}(s)$ isnot identically zero. As such, we have an orthogonal frame $\{\mathbf{T}, \mathbf{N}, \mathbf{B}\}$ described by

$$
\mathbf{T}=\frac{d \beta}{d s}, \mathbf{N}=\frac{d \mathbf{T}}{d s}, \mathbf{B}=\mathbf{T} \times \mathbf{N} .
$$


The relations between those and the classical Frenet frame $\{\mathbf{t}, \mathbf{n}, \mathbf{b}\}$ at non-zero points of $\kappa$ are

$$
\begin{aligned}
& \mathbf{T}=\mathbf{t}, \\
& \mathbf{N}=\kappa \mathbf{n}, \\
& \mathbf{B}=\kappa \mathbf{b} .
\end{aligned}
$$

Thus, $\mathbf{N}\left(s_{0}\right)=\mathbf{B}\left(s_{0}\right)=0$ when $\kappa\left(s_{0}\right)=0$ and squares of the length of $\mathbf{N}$ and $\mathbf{B}$ vary analytically in s. By the definition of $\{\mathbf{T}, \mathbf{N}, \mathbf{B}\}$ or Equation (5), a simple calculation shows that

$$
\begin{aligned}
& \mathbf{T}^{\prime}(s)=\mathbf{N}, \\
& \mathbf{N}^{\prime}(s)=-\kappa^{2} \mathbf{T}+\frac{\kappa^{\prime}}{\kappa} \mathbf{N}+\tau \mathbf{B}, \\
& \mathbf{B}^{\prime}(s)=-\tau \mathbf{N}+\frac{\kappa^{\prime}}{\kappa} \mathbf{B},
\end{aligned}
$$

where

$$
\tau(s)=\frac{\operatorname{det}\left(\alpha^{\prime}, \alpha^{\prime \prime}, \alpha^{\prime \prime \prime}\right)}{\kappa^{2}},
$$

The Equation (6) corresponds to the Frenet-Serret equation in the classical case. Moreover, $\{\mathbf{T}, \mathbf{N}, \mathbf{B}\}$ satisfies:

$$
\begin{aligned}
& g(\mathbf{T}, \mathbf{T})=1, g(\mathbf{N}, \mathbf{N})=g(\mathbf{B}, \mathbf{B})=\kappa^{2}, \\
& g(\mathbf{T}, \mathbf{N})=g(\mathbf{T}, \mathbf{B})=g(\mathbf{N}, \mathbf{B})=0,
\end{aligned}
$$

where $g($,$) denotes the inner product of \mathrm{E}^{3}$. We note that the essential quantities in Equations (6) and (7) are $\kappa^{2}$ and $\tau(s)$ which are analytic in $s$ [26-28].

The unit normal vector field $N$ be on a surface $P$ defined by

$$
N=\frac{P_{s} \wedge P_{\theta}}{\left\|P_{s} \wedge P_{\theta}\right\|}
$$

where $P_{s}=\frac{\partial P(s, \theta)}{\partial s}$. Also, the first $I$ and second fundamental forms $I I$ of the surface $P$ are defined by

$$
\begin{aligned}
& I=E d s^{2}+2 F d s d \theta+G d \theta^{2}, \\
& I I=e d s^{2}+2 f d s d \theta+g d \theta^{2},
\end{aligned}
$$

where

$$
\begin{aligned}
& E=\left\langle P_{s}, P_{s}\right\rangle, F=\left\langle P_{s}, P_{\theta}\right\rangle, G=\left\langle P_{\theta}, P_{\theta}\right\rangle, \\
& e=\left\langle P_{s s}, N\right\rangle, f=\left\langle P_{s \theta}, N\right\rangle, g=\left\langle P_{\theta \theta}, N\right\rangle .
\end{aligned}
$$

Additionally, the invariant functions of the Gaussian curvature $K$ and the mean curvature $H$ of the surface are described by

$$
\begin{aligned}
& K=\frac{e g-f^{2}}{E G-F^{2}}, \\
& H=\frac{E g-2 F f+G e}{2\left(E G-F^{2}\right)},
\end{aligned}
$$

respectively.

Definition 2.1. [29] Let first fundamental form $\{E, F, G\}$ of a surface evolution satisfies following,

$$
\frac{\partial E}{\partial t}=\frac{\partial F}{\partial t}=\frac{\partial G}{\partial t}=0
$$

Accordingly, the surface evolution $P(s, v, t)$ and its flow $\frac{\partial P}{\partial t}$ are inextensible. 


\section{New Modified Roller Coaster Surface in Space}

In this section, we introduce the first new modified roller coaster surface by rotating the orthogonal modified frame. We also express this new surface and its properties. In addition, we obtain new roller coaster surfaces by translating the orthogonal modified frame. As a result, these new roller coaster surfaces are called new modified roller coaster surfaces.

To separate a roller coaster surface according to the modified orthogonal frame from that of the Frenet-Serret frame, in the rest of the paperwe shall use notation as a modified roller coaster surface.

In terms of the modified orthogonal frame $(\mathbf{T}(s), \mathbf{N}(s), \mathbf{B}(s))$ the parametric expression of the modified roller coaster surfaces can be given by Equation (5)

$$
P(s, v)=\beta(s)+r\left(\cos v \mathbf{T}(s)+\frac{\sin v}{\kappa}(\cos \varphi(s) \mathbf{N}(s)+\sin \varphi(s) \mathbf{B}(s))\right) .
$$

Proposition 3.1. In terms of the modified orthogonal frame $(\mathbf{T}(s), \mathbf{N}(s), \mathbf{B}(s)$ ), the parametric expression of the modified roller coaster surfaces can be given as follows:

$$
\begin{aligned}
& K=\frac{1}{\Gamma \eta}\left(\lambda _ { 1 } \left(\left[(1-r \kappa \sin v \cos \varphi)^{\prime}+r \kappa \sin v \varphi^{\prime} \sin \varphi-r \kappa^{2} \cos v\right.\right.\right. \\
& +r \kappa \tau \sin v \sin \varphi]+\kappa^{2} \lambda_{2}\left[1-r \sin v \kappa \cos \varphi+\left(r \cos v-\frac{r \varphi^{\prime}}{\kappa} \sin v \sin \varphi\right.\right. \\
& \left.-\frac{r \tau}{\kappa} \sin v \sin \varphi\right)^{\prime}+\frac{r \kappa^{\prime}}{\kappa} \cos v-\sin v \frac{r \kappa^{\prime} \varphi^{\prime}}{\kappa^{2}} \sin \varphi-\frac{r \kappa^{\prime} \tau}{\kappa^{2}} \sin v \sin \varphi \\
& \left.-\frac{r \tau^{2}}{\kappa} \sin v \cos \varphi-\frac{r \tau \varphi^{\prime}}{\kappa} \cos \varphi \sin v\right]+\kappa^{2} \lambda_{3}\left[r \tau \cos v-\frac{r \tau \varphi^{\prime}}{\kappa} \sin v \sin \varphi\right. \\
& -\frac{r \tau^{2}}{\kappa} \sin v \sin \varphi+\left(\sin v \frac{r \tau}{\kappa} \cos \varphi+\frac{r \varphi^{\prime}}{\kappa} \cos \varphi \sin v\right)^{\prime}+\frac{r \kappa^{\prime} \tau}{\kappa^{2}} \sin v \cos \varphi \\
& \left.\left.+\frac{r \kappa^{\prime} \varphi^{\prime}}{\kappa^{2}} \cos \varphi \sin v\right]\right)\left(-r \lambda_{1} \cos v-r \sin v \lambda_{2} \kappa \cos \varphi-r \lambda_{3} \kappa \sin v \sin \varphi\right) \\
& -\frac{1}{\eta \Gamma^{2}}\left(\left[-r \kappa \lambda_{1} \cos v \cos \varphi\right]+\left[-r \kappa^{2} \lambda_{2} \sin v-r \kappa \lambda_{2} \varphi^{\prime} \cos v \sin \varphi\right.\right. \\
& \left.\left.-r \kappa \tau \lambda_{2} \cos v \sin \varphi\right]+\left[r \kappa \lambda_{3} \varphi^{\prime} \cos \varphi \cos v+r \tau \lambda_{3} \cos v \cos \varphi\right]\right)^{2},
\end{aligned}
$$

where

$$
\begin{aligned}
& \lambda_{1}=\frac{r^{2}}{\kappa} \cos ^{2} v \sin \varphi-\frac{r^{2} \varphi^{\prime}}{\kappa^{2}} \cos v \sin v \\
& -\frac{r^{2} \tau}{\kappa^{2}} \cos v \sin v, \\
& \lambda_{2}=-\frac{r}{\kappa} \cos v \sin \varphi+\cos v r^{2} \sin v \cos \varphi \sin \varphi \\
& -\frac{r^{2} \tau}{\kappa} \sin ^{2} v \cos \varphi-\frac{r^{2} \varphi^{\prime}}{\kappa} \cos \varphi \sin ^{2} v, \\
& \lambda_{3}=\frac{r}{\kappa} \cos v \cos \varphi+r^{2} \cos v \sin v \sin ^{2} \varphi \\
& -\frac{r^{2} \tau}{\kappa} \sin ^{2} v \sin \varphi-\frac{r^{2} \varphi^{\prime}}{\kappa} \sin ^{2} v \sin \varphi, \\
& \eta=\left[r-r^{2} \kappa \sin v \cos \varphi\right]^{2}+\left[r^{2} \kappa \cos v\right. \\
& \left.-r^{2} \varphi^{\prime} \sin v \sin \varphi-r^{2} \tau \sin v \sin \varphi\right]^{2} \\
& +\left[r^{2} \tau \sin v \cos \varphi+r^{2} \varphi^{\prime} \cos \varphi \sin v\right]^{2} \\
& -\left[-r \sin v+r^{2} \kappa \cos \varphi\right]^{2} \\
& \lambda_{1}=\frac{r^{2}}{\kappa} \cos { }^{2} v \sin \varphi-\frac{r^{2} \varphi^{\prime}}{\kappa^{2}} \cos v \sin v \\
& -\frac{r^{2} \tau}{\kappa^{2}} \cos v \sin v, \\
& \lambda_{2}=-\frac{r}{\kappa} \cos v \sin \varphi+\cos v r^{2} \sin v \cos \varphi \sin \varphi \\
& -\frac{r^{2} \tau}{\kappa} \sin { }^{2} v \cos \varphi-\frac{r^{2} \varphi^{\prime}}{\kappa} \cos \varphi \sin { }^{2} v, \\
& \lambda_{3}=\frac{r}{\kappa} \cos v \cos \varphi+r^{2} \cos v \sin v \sin ^{2} \varphi \\
& -\frac{r^{2} \tau}{\kappa} \sin v \sin \varphi-\frac{r^{2} \varphi^{\prime}}{\kappa} \sin { }^{2} v \sin \varphi, \\
& \eta=\left[r-r^{2} \kappa \sin v \cos \varphi\right]^{2}+\left[r^{2} \kappa \cos v\right. \\
& \left.-r^{2} \varphi^{\prime} \sin v \sin \varphi-r^{2} \tau \sin v \sin \varphi\right]^{2} \\
& +\left[r^{2} \tau \sin v \cos \varphi+r^{2} \varphi^{\prime} \cos \varphi \sin v\right]^{2} \\
& -\left[-r \sin v+r^{2} \kappa \cos \varphi\right]^{2}
\end{aligned}
$$


and

$$
\Gamma=\sqrt{\begin{array}{c}
{\left[\frac{r^{2}}{\kappa} \cos ^{2} v \sin \varphi-\frac{r^{2} \varphi^{\prime}}{\kappa^{2}} \cos v \sin v-\frac{r^{2} \tau}{\kappa^{2}} \cos v \sin v\right]^{2}} \\
\left.+r \cos v \sin \varphi+r^{2} \kappa \cos v \sin v \cos \varphi \sin \varphi-r^{2} \tau \sin ^{2} v \cos \varphi-r^{2} \varphi^{\prime} \cos \varphi \sin ^{2} v\right]^{2} \\
+\left[r \cos v \cos \varphi+r^{2} \kappa \cos v \sin v \sin ^{2} \varphi-r^{2} \tau \sin ^{2} v \sin \varphi-r^{2} \varphi^{\prime} \sin ^{2} v \sin \varphi\right]^{2} .
\end{array}}
$$

Proof. We have the natural frame $\left\{P_{s}, P_{v}\right\}$ of the surface $P(s, v)$ given by

$$
\begin{aligned}
& P_{S}=[1-r \kappa \sin v \cos \varphi] \mathbf{T}+[r \cos v \\
& +r \sin v\left(\frac{\cos \varphi}{\kappa}\right)^{\prime}+\frac{r \kappa^{\prime}}{\kappa^{2}} \sin v \cos \varphi \\
& \left.-\frac{r \tau}{\kappa} \sin v \sin \varphi\right] \mathbf{N}+\left[\frac{r \tau}{\kappa} \sin v \cos \varphi\right. \\
& \left.+r \sin v\left(\frac{\sin \varphi}{\kappa}\right)^{\prime}+\frac{r \kappa^{\prime}}{\kappa^{2}} \sin v \sin \varphi\right] \mathbf{B}, \\
& P_{v}=[-r \sin v] \mathbf{T}+\left[\frac{r}{\kappa} \cos v \cos \varphi\right] \mathbf{N} \\
& +\left[\frac{r}{\kappa} \cos v \sin \varphi\right] \mathbf{B} .
\end{aligned}
$$

The quantities $E, F, G$ are calculated to be

$$
\begin{aligned}
& E=[1-r \kappa \sin v \cos \varphi]^{2} \\
& +\left[-r \varphi^{\prime} \sin v \sin \varphi+r \kappa \cos v-r \tau \sin v \sin \varphi\right]^{2} \\
& +\left[r \varphi^{\prime} \cos \varphi \sin v+r \tau \sin v \cos \varphi\right]^{2}, \\
& F=-r \sin v+r^{2} \kappa \cos \varphi, \\
& G=r^{2} .
\end{aligned}
$$

The standard unit normal vector field $N$ given by

$$
N=\frac{1}{\Gamma}\left(\lambda_{1} \mathbf{T}+\lambda_{2} \mathbf{N}+\lambda_{3} \mathbf{B}\right)
$$

where

$$
\begin{aligned}
& \lambda_{1}=\frac{r^{2}}{\kappa} \cos ^{2} v \sin \varphi-\frac{r^{2} \varphi^{\prime}}{\kappa^{2}} \cos v \sin v-\frac{r^{2} \tau}{\kappa^{2}} \cos v \sin v, \\
& \lambda_{2}=-\frac{r}{\kappa} \cos v \sin \varphi+r^{2} \cos v \sin v \cos \varphi \sin \varphi-\frac{r^{2} \tau}{\kappa} \sin ^{2} v \cos \varphi-\frac{r^{2} \varphi^{\prime}}{\kappa} \cos \varphi \sin ^{2} v, \\
& \lambda_{3}=\frac{r}{\kappa} \cos v \cos \varphi+r^{2} \cos v \sin v \sin ^{2} \varphi-\frac{r^{2} \tau}{\kappa} \sin ^{2} v \sin \varphi-\frac{r^{2} \varphi^{\prime}}{\kappa} \sin ^{2} v \sin \varphi,
\end{aligned}
$$

and

$$
\Gamma=\sqrt{\begin{array}{c}
{\left[\frac{r^{2}}{\kappa} \cos ^{2} v \sin \varphi-\cos v \frac{r^{2} \varphi^{\prime}}{\kappa^{2}} \sin v-\cos v \frac{r^{2} \tau}{\kappa^{2}} \sin v\right]^{2}} \\
\left.+-r \cos v \sin \varphi+r^{2} \cos v \kappa \sin v \cos \varphi \sin \varphi-r^{2} \tau \sin ^{2} v \cos \varphi-r^{2} \varphi^{\prime} \cos \varphi \sin ^{2} v\right]^{2} \\
+\left[r \cos v \cos \varphi+r^{2} \cos v \kappa \sin v \sin ^{2} \varphi-r^{2} \tau \sin ^{2} v \sin \varphi-r^{2} \varphi^{\prime} \sin ^{2} v \sin \varphi\right]^{2}
\end{array}}
$$


Components of second fundamental form are

$$
\begin{aligned}
& e=\frac{\lambda_{1}}{\Gamma}\left(\left[(1-r \kappa \sin v \cos \varphi)^{\prime}-r \kappa^{2} \cos v+r \kappa \varphi^{\prime} \sin v \sin \varphi+r \kappa \tau \sin v \sin \varphi\right]\right. \\
& +\frac{\kappa^{2} \lambda_{2}}{\Gamma}\left[1-r \kappa \sin v \cos \varphi+\left(r \cos v-\frac{r \varphi^{\prime}}{\kappa} \sin v \sin \varphi-\frac{r \tau}{\kappa} \sin v \sin \varphi\right)^{\prime}\right. \\
& +\frac{r \kappa^{\prime}}{\kappa} \cos v-\sin v \frac{r \kappa^{\prime} \varphi^{\prime}}{\kappa^{2}} \sin \varphi-\sin v \frac{r \kappa^{\prime} \tau}{\kappa^{2}} \sin \varphi \\
& \left.-\frac{r \tau^{2}}{\kappa} \sin v \cos \varphi-\frac{r \tau \varphi^{\prime}}{\kappa} \cos \varphi \sin v\right]+\frac{\kappa^{2} \lambda_{3}}{\Gamma}[r \tau \cos v \\
& -\frac{r \tau \varphi^{\prime}}{\kappa} \sin v \sin \varphi-\frac{r \tau^{2}}{\kappa} \sin v \sin \varphi+\left(\cos \varphi \frac{r \tau}{\kappa} \sin v+\cos \varphi \frac{r \varphi^{\prime}}{\kappa} \sin v\right)^{\prime} \\
& \left.+\frac{r \kappa^{\prime} \tau}{\kappa^{2}} \sin v \cos \varphi+\frac{r \kappa^{\prime} \varphi^{\prime}}{\kappa^{2}} \cos \varphi \sin v\right], \\
& f=\frac{\lambda_{1}}{\Gamma}[-r \cos v \kappa \cos \varphi]+\frac{\lambda_{2}}{\Gamma}\left[-r \kappa^{2} \sin v-r \kappa \cos v \varphi^{\prime} \sin \varphi\right. \\
& -r \cos v \kappa \tau \sin \varphi]+\frac{\lambda_{3}}{\Gamma}\left[r \tau \cos v \cos \varphi+r \kappa \varphi^{\prime} \cos \varphi \cos v\right], \\
& g=-\frac{\lambda_{1}}{\Gamma}[r \cos v]-\frac{\lambda_{2}}{\Gamma}[r \kappa \sin v \cos \varphi]-\frac{\lambda_{3}}{\Gamma}[r \kappa \sin v \sin \varphi] .
\end{aligned}
$$

On the other hand,

$$
\begin{aligned}
& \eta=\left[r-r^{2} \sin v \kappa \cos \varphi\right]^{2}+\left[r^{2} \kappa \cos v\right. \\
& \left.-r^{2} \sin \varphi \varphi^{\prime} \sin v-r^{2} \sin \varphi \tau \sin v\right]^{2} \\
& +\left[r^{2} \tau \sin v \cos \varphi+r^{2} \varphi^{\prime} \cos \varphi \sin v\right]^{2} \\
& -\left[r^{2} \kappa \cos \varphi-r \sin v\right]^{2} .
\end{aligned}
$$

So, the proof is complete.

Corollary 3.2. Assume the spine curve of a modified roller coaster surface is a unit speed curve $\beta: I \rightarrow \mathrm{E}^{3}$ with nonzero curvature. Then, the mean curvature of modified roller coaster surface $P(s, v)$ is given by

$$
\begin{aligned}
& H=\frac{1}{2 \eta}\left(\left([1-r \kappa \sin v \cos \varphi]^{2}+\left[r \kappa \cos v-r \varphi^{\prime} \sin v \sin \varphi\right.\right.\right. \\
& \left.-r \tau \sin v \sin \varphi]^{2}+\left[r \tau \sin v \cos \varphi+r \varphi^{\prime} \cos \varphi \sin v\right]^{2}\right)\left(\frac { - 1 } { \Gamma } \left(r \lambda_{1} \cos v\right.\right. \\
& \left.-r \kappa \lambda_{2} \sin v \cos \varphi-r \kappa \lambda_{3} \sin v \sin \varphi\right)-\frac{2}{\Gamma}\left(-r \sin v+r^{2} \kappa \cos \varphi\right)\left(-r \kappa \lambda_{1} \cos v \cos \varphi\right. \\
& -r \kappa^{2} \lambda_{2} \sin v-r \kappa \lambda_{2} \varphi^{\prime} \cos v \sin \varphi-r \kappa \tau \lambda_{2} \cos v \sin \varphi+r \tau \lambda_{3} \cos v \cos \varphi \\
& \left.+r \kappa \lambda_{3} \varphi^{\prime} \cos \varphi \cos v\right)+\frac{r^{2}}{\Gamma}\left(\lambda_{1}(1-r \kappa \sin v \cos \varphi)^{\prime}-r \kappa^{2} \lambda_{1} \cos v\right. \\
& +r \kappa \varphi^{\prime} \lambda_{1} \sin v \sin \varphi+r \kappa \tau \lambda_{1} \sin v \sin \varphi+\kappa^{2} \lambda_{2}-r \kappa^{3} \lambda_{2} \sin v \cos \varphi \\
& +\kappa^{2} \lambda_{2}\left(r \cos v-\frac{r \varphi^{\prime}}{\kappa} \sin v \sin \varphi-\frac{r \tau}{\kappa} \sin v \sin \varphi\right)^{\prime}+r \kappa^{\prime} \kappa \lambda_{2} \cos v \\
& -r \kappa^{\prime} \varphi^{\prime} \lambda_{2} \sin v \sin \varphi-r \kappa^{\prime} \tau \lambda_{2} \sin v \sin \varphi+\left[r \tau \kappa^{2} \lambda_{3} \cos v-r \tau \varphi^{\prime} \kappa \lambda_{3} \sin v \sin \varphi\right. \\
& -r \tau^{2} \kappa \lambda_{3} \sin v \sin \varphi+\kappa^{2} \lambda_{3}\left(\frac{r \tau}{\kappa} \sin v \cos \varphi+\frac{r \varphi^{\prime}}{\kappa} \cos \varphi \sin v\right)^{\prime}+r \kappa^{\prime} \tau \kappa \lambda_{3} \sin v \cos \varphi \\
& \left.\left.+r \kappa \kappa^{\prime} \varphi^{\prime} \lambda_{3} \cos \varphi \sin v\right)\right) .
\end{aligned}
$$

Corollary 3.3. Assume the spine curve of a modified roller coaster surface is a unit speed curve $\gamma: I \rightarrow \mathrm{E}^{3}$ with nonzero curvature. If $\frac{\partial \mathrm{P}}{\partial t}$ is inextensible, then

$$
\begin{aligned}
& \frac{\partial}{\partial t}\left((1-r \kappa \sin v \cos \varphi)^{2}+\left(r \kappa \cos v-r \varphi^{\prime} \sin v \sin \varphi\right.\right. \\
& \left.-r \tau \sin v \sin \varphi)^{2}+\left(r \tau \sin v \cos \varphi+r \varphi^{\prime} \cos \varphi \sin v\right)^{2}\right)=0, \\
& \frac{\partial}{\partial t}\left(-r \sin v+r^{2} \kappa \cos \varphi\right)=0, \\
& \frac{\partial}{\partial t}\left(r^{2}\right)=0 .
\end{aligned}
$$

Corollary 3.4. Let a modified roller coaster surface $P(s, v)$ and $\bar{P}(s, v)$ be a parallel surface of $P(s, v)$ in Euclidean 3-space. Then, the equation of $\bar{P}(s, v)$ is givenby

$$
\begin{gathered}
\bar{P}(s, v)=\beta(s)+r\left(\cos v \mathbf{T}(s)+\frac{\sin v}{\kappa}(\cos \varphi(s) \mathbf{N}(s)\right. \\
\quad+\sin \varphi(s) \mathbf{B}(s)))+\delta\left(\frac{1}{\Gamma}\left(\lambda_{1} \mathbf{T}+\lambda_{2} \mathbf{N}+\lambda_{3} \mathbf{B}\right),\right.
\end{gathered}
$$


where $\delta$ is a constant.

\section{Application}

First, let us regarda unit speed circular helix by

$$
\beta=\beta(s)=\left(a \cos \frac{s}{d}, a \sin \frac{s}{d}, \frac{b s}{d}\right),
$$

where $d=\sqrt{a^{2}+b^{2}} \in R$. By using this curve, we obtain a new modified roller coaster surface and its parallel surface:

For $\varphi=\frac{\pi}{8}, v=\frac{\pi}{4}, r=\frac{1}{2}$ : Figures 1 and 2.



Figure 1. Modified roller coaster surface.

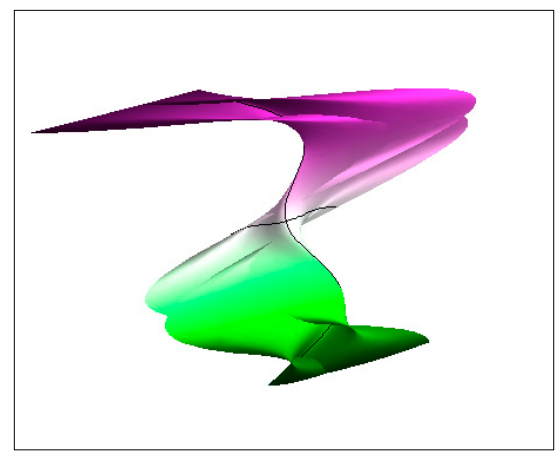

Figure 2. Its parallel surface.

For $\varphi=\frac{\pi}{3}, v=\frac{\pi}{3}, r=2$ : Figures 3 and 4 .

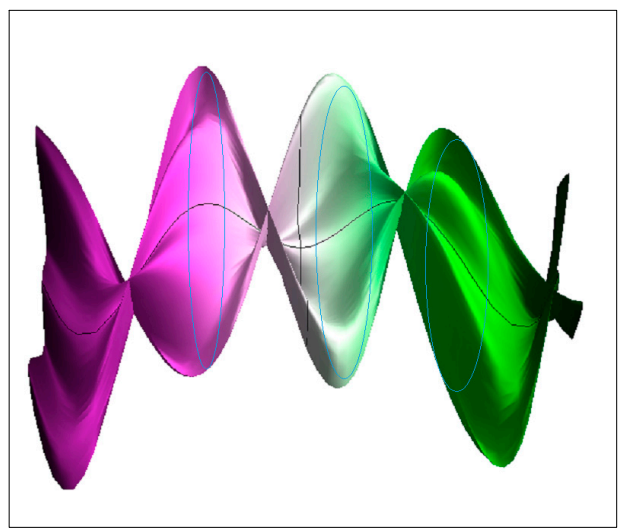

Figure 3. Modified roller coaster surface. 


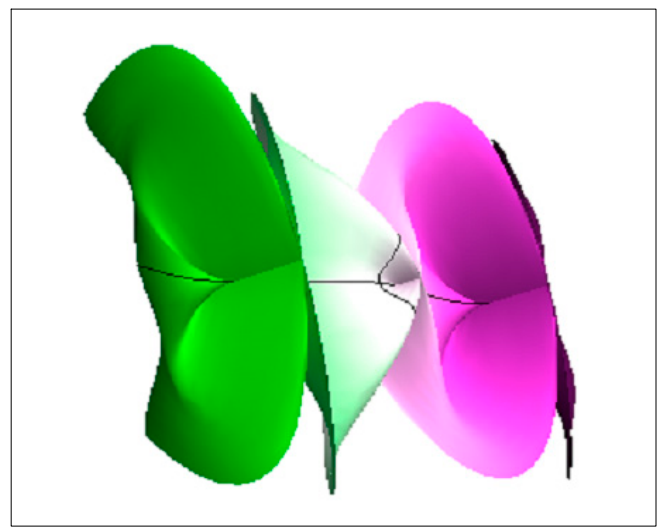

Figure 4. Its parallel surface.

For $\varphi=-\frac{\pi}{3}, v=\frac{\pi}{3}, r=4$ : Figures 5 and 6 .

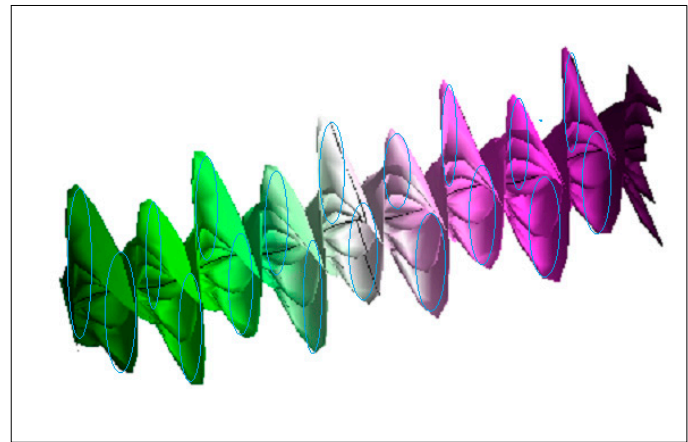

Figure 5. Modified roller coaster surface.

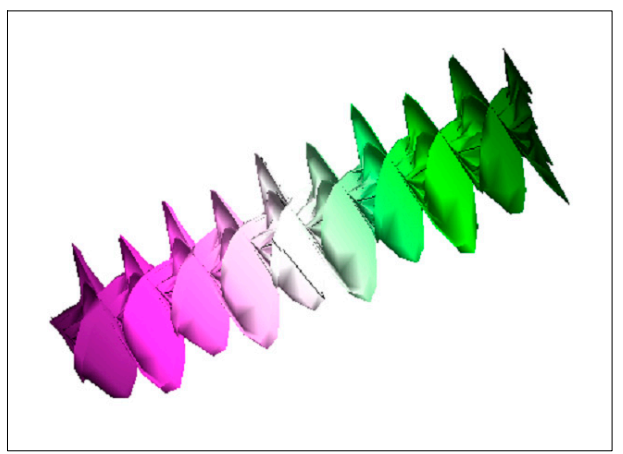

Figure 6. Its parallel surface.

\section{Conclusions}

In the literature, we examined the surface of a roller coaster, which has been been studied extensively. The surface of a roller coaster is a similar surface to the tangent surface that can be developed.

In this paper, new roller coaster surfaces according to the modified orthogonal frame are introduced in Euclidean 3-space. Results are provided in relationshipto these new modified roller coaster surfaces. In addition, these concepts are applied to exampleswith figures.

As a result, we obtain new roller coaster surfaces by translating the orthogonal modified frame. Accordingly, these new roller coaster surfaces are called new modified roller coaster surfaces.

According to this method, a new roller coaster is modeled. In light of these results, we will study this concept in the Minkowski space. 
Author Contributions: Conceptualization, S.B. and T.K.; Methodology, S.B. and T.K.; Software, S.B. and T.K.; Validation, S.B. and T.K.; Formal Analysis, S.B. and T.K.; Investigation, S.B. and T.K.; Resources, S.B. and T.K.; Data Curation, S.B. and T.K.; Writing-Original Draft Preparation, S.B. and T.K.; Writing-Review \& Editing, S.B. and T.K.; Visualization, S.B. and T.K.; Supervision, S.B. and T.K.; Project Administration, S.B. and T.K.; Funding Acquisition, S.B. and T.K.

Funding: This research received no external funding.

Conflicts of Interest: The authors declare no conflicts of interest.

\section{References}

1. Zhang, X.; Zhang, J.; Pang, B.; Zhao, W. An accurate prediction method of cutting forces in 5-axis flank milling of sculptured surface. Int. J. Mach. Tool. Manuf. 2016, 104, 26-36. [CrossRef]

2. Ryuh, B.S.; Pennock, G.R. Accurate Motion of a Robot End-Effector Using the Curvature Theory of Ruled Surfaces. J. Mech. Transm. Autom. Des. 1988, 110, 383-388. [CrossRef]

3. Li, C.Y.; Wang, R.H.; Zhu, C.G. An approach for designing a developable surface through a given line of curvature. Comput. Aided Des. 2013, 45, 621-627. [CrossRef]

4. Pottmann, H.; Wallner, J. Computational Line Geometry; Springer: Berlin, Germany, 2000.

5. Sprott, K.; Ravani, B. Cylindrical milling of ruled surfaces. Int. J. Adv. Manuf. Technol. 2008, 38, 649-656. [CrossRef]

6. Izumiya, S.; Takeuchi, N. Special curves and ruled surfaces. Contrib. Algebra Geom. 2003, 44, $203-212$.

7. Baş, S.; Asil, V.; Körpınar, T. A New Version of Five-Axis Motion of Spheres with Spacelike Curves in Minkowski Space. J. Adv. Phys. 2018, 7, 366-375.

8. Webster, R.J.; Jones, B.A. Design and kinematic modeling of constant curvature continuum robots. Int. J. Robot. Res. 2010, 29, 1661-1683. [CrossRef]

9. Marsh, D. Applied Geometry For Computer Graphics And CAD; Springer Undergraduate Mathematics Series: London, UK, 2005.

10. Bükcü, B.; Karacan, M.K. An alternative moving frame for tubular surfaces around timelike curves in the Minkowski 3-space. Balk. J. Geom. Appl. 2007, 12, 73-80.

11. Dogan, F.; Yayl1, Y. On the Curvatures of Tubular Surfaces with Bishop Frame. Commun. Fac. Sci. Univ. Ank. Series A1 2011, 60, 59-69.

12. Karacan, M.K.; Es, H.; Yaylı, Y. Singuler Points of Tubular Surface in Minkowski Surfaces. Sarajevo J. Math. 2006, 2, 73-82.

13. Lu, W.; Pottmann, H. Pipe Surfaces With Rational Spine Curve Are Rational. Comput. Aided Geom. Des. 1996, 13, 621-628. [CrossRef]

14. Körpınar, T.; Turhan, E. On characterization of $B$-canal surfaces in terms of biharmonic $B$-slant helices according to Bishop frame in Heisenberg group Heis ${ }^{3}$. J. Math. Anal. Appl. 2012, 382, 57-65. [CrossRef]

15. Körpınar, T.; Turhan, E. Time-Canal Surfaces Around Biharmonic Particles and Its Lorentz Transformations in Heisenberg space-time. Int. J. Theor. Phys. 2014, 53, 1502-1520. [CrossRef]

16. Xu, Z.; Feng, R.; Sun, J.G. Analytic and algebraic properties of canal surfaces. J. Comput. Appl. Math. 2006, 195, 220-228.

17. Maurer, C. Applications of Sphere Geometry in Canal Surface Design, in Curves and Surfaces; Laurent, P.J., Rabut, C., Schumaker, L.L., Eds.; Vanderbilt University Press: Nashville, TN, USA, 2000.

18. Stasiak, A.; Maddocks, J.H. Best Packing in Proteins and DNA. Nat. Lond. 2000, 406, 251-253. [CrossRef] [PubMed]

19. Hunt, K.H. Kinematic Geometry of Mechanisms; Clarendon: Oxford, UK, 1978.

20. Cui, L.; Wang, D.; Dai, J.S. Kinematic Geometry of Circular Surfaces With a Fixed Radius Based on Euclidean Invariants. J. Mech. Des. 2009, 131. [CrossRef]

21. Zahariev, E.; Cuadrado, J. IUTAM Symposium on Intelligent Multibody Systems Dynamics, Control, Simulation; Springer Nature Switzerland AG: Basel, Switzerland, 2019.

22. Izumiya, S.; Saji, S.; Takeuchi, N. Circular surfaces. Adv. Geom. 2005, 7, 295-313. [CrossRef]

23. Çanakc1, Z.; Tuncer, O.O.; Gök, I.; Yaylı, Y. The construction of circular surfaces with quaternions. Asian Eur. J. Math. 2019, 12. [CrossRef] 
24. Tuncer, O.O.; Çanakc1, Z.; Gök, I.; Yaylı, Y. Circular Surfaces with Split Quaternionic Representations in Minkowski 3-space. Adv. Appl. Clifford Algebras 2018, 28. [CrossRef]

25. Abdel-Baky, R.A.; Unlutürk, Y. On the curvatures of spacelike circular surfaces. Kuwait J. Sci. 2016, 43, 50-58.

26. Bükcü, B.; Karacan, M.K. Spherical Curves with Modified Orthogonal Frame. J. New Result. Sci. 2016, 10, 60-68.

27. Bükcü, B.; Karacan, M.K. On The Modified Orthogonal Frame with Curvature and Torsion in 3-Space. Math. Sci. Appl. E. Note. 2016, 4, 184-188.

28. Sasai, T. The Fundamental Theorem of Analytic Space Curves And Apparent Singularities of Fuchsian Differential Equations. Tohoku Math. J. 1984, 36, 17-24. [CrossRef]

29. Kwon, D.Y.; Park, F.C.; Chi, D.P. Inextensible flows of curves and developable surfaces. Appl. Math. Lett. 2005, 18, 1156-1162.

(C) 2019 by the authors. Licensee MDPI, Basel, Switzerland. This article is an open access article distributed under the terms and conditions of the Creative Commons Attribution (CC BY) license (http://creativecommons.org/licenses/by/4.0/). 